\title{
Perfil socioeconômico dos feirantes e consumidores da Feira do Produtor de Passo Fundo, RS
}

\author{
Socioeconomic profile of farmers and consumers of the Passo Fundo farmer's \\ market, Rio Grande de Sul state, Brazil
}

\author{
Hélio Carlos Rocha ${ }^{\mathrm{I}}$ Carlos Costa ${ }^{\mathrm{II}}$ Florindo Luiz Castoldi ${ }^{\mathrm{I}}$ Dileta Cecchetti \\ Eunice de Oliveira Calvete ${ }^{\mathrm{I}}$ Betânia dos Santos Lodi' ${ }^{\mathrm{III}}$
}

\section{RESUMO}

A Feira do Produtor de Passo Fundo, embora seja um evento institucionalizado há 22 anos, ainda não havia sido objeto de estudo formalizado quanto à caracterização social e econômica de quem nela vende e compra. O objetivo deste trabalho foi traçar o perfil socioeconomico dos feirantes e consumidores da Feira do Produtor de Passo Fundo, RS. Os dados foram obtidos por meio da aplicação de questionário junto a 58 feirantes e 471 consumidores. Os questionários abrangeram questões referentes aos aspectos socioeconômicos dos entrevistados $e$ produtos ofertados/comprados na feira, como hortaliças, frutas, grãos, produtos de origem animal e produtos artesanais. Verificou-se que a faixa etária dos consumidores concentra-se entre 35 a 65 anos. Os feirantes apresentam idade média entre 40 e 55 anos. Um terço dos feirantes possuem núcleo familiar composto por até três pessoas. $O$ nivel de escolaridade mais comum neste grupo é o fundamental. Aproximadamente $80 \%$ dos entrevistados relataram ganho mensal de até $R \$ 1.000,00$. Os consumidores são, em sua maioria, indivíduos das classes B2, C e D, dos quais $60 \%$ declararam renda mensal entre $R \$ 500,00$ e $R \$ 2.000,00$. O valor médio de consumo foi de $R \$ 25,94$ feir $^{-1}$. Os homens gastaram, em média, R\$26,34 e as mulheres $R \$ 25,48$ feira $^{-1}$. As hortaliças e as frutas constituem mais da metade (59\%) dos itens comprados na Feira em análise.

Palavras-chave: feira livre, caracterização socioeconômica, rentabilidade feirante, consumo feira livre.

\section{ABSTRACT}

Though the Passo Fundo farmer's market has been in existed for over 22 years, the social and economic

\begin{abstract}
status of farmers and consumers attended has not been documented yet. Consequently, this study sought to provide the first detailed socioeconomic profile of farmers and consumers that participate in the market. Questionnaires were administered to 58 farmers that hold permanent stalls at the market and another to 471 consumers. The questionnaires had queries regarding the socioeconomic aspects of farmers and consumers, and products offered/purchased at the market, such as, vegetables, fruits, grains, products of animal origin, and handicrafts. While most consumers ranged from 35 to 65 years of age, farmers tended to be between 40 and 55 years old. One-third of farmers had a familiar nucleus composed of up to three people and most had completed elementary school. Nearly $80 \%$ of farmers reported a monthly income of under 1,000 Brazilian Reais (R\$), or roughly $\$ 620$ US. Typical consumers of the Passo Fundo Farmer's market are of the middle to lower (B2, C and D) social classes. Roughly $60 \%$ of them declared monthly incomes between 500 and $2000 R \$$ (\$310-1240 US). The average consumption was $R \$ 25.94$ farmer-1 $^{-1}$ (\$16.11 US), with men's mean purchases ( $\$ 26.34$ farmer $^{-1}, \$ 16.41$ US) being slightly greater than for women $\left(R \$ 25.48\right.$ farmer $^{-1}, \$ 15.83$ US). Vegetables and fruits made up $59 \%$ of the items purchased by consumers.
\end{abstract}

Key words: farmer's market, Brazil, socioeconomic status, farmer's profitability, farmer to consumer transaction.

\section{INTRODUÇÃO}

A busca por alimentos frescos, presumivelmente cultivados sem o uso, ou com uso menos intensivo, de defensivos agrícolas e preços

\footnotetext{
IFaculdade de Agronomia e Medicina Veterinária da Universidade de Passo Fundo (UPF), 99001-970, CP 6111, Passo Fundo, RS, Brasil. E-mail: helio@upf.br. Autor para correspondência.

${ }^{\mathrm{II}}$ Curso de Administração da Faculdade IDEAU, Getúlio Vargas, RS, Brasil.

${ }^{\mathrm{III} N u ́ c l e o ~ d e ~ A g r i c u l t u r a ~ d a ~ S e c r e t a r i a ~ d o ~ I n t e r i o r ~ d a ~ P r e f e i t u r a ~ M u n i c i p a l ~ d e ~ P a s s o ~ F u n d o, ~ P a s s o ~ F u n d o, ~ R S, ~ B r a s i l . ~}$
} 
mais acessíveis são os atrativos que levam muitos consumidores a preferirem as feiras livres aos mercados tradicionais. As feiras, que normalmente ocorrem em áreas abertas, são os locais onde os produtores, geralmente pequenos agricultores, comercializam, principalmente, frutas e hortaliças. A cidade de Passo Fundo, RS, sedia feiras de produtores que se diferenciam pela suas dimensões. A feira de produtor objeto deste estudo acontece regularmente na antiga estação ferroviária de Passo Fundo (Gare). É a mais importante, tanto pelo número de feirantes, que ocupam $75 \%$ da área do pavilhão, como pela quantidade de consumidores.

A citação mais antiga encontrada sobre a Feira do Produtor de Passo Fundo data de 1ㅇ de agosto de 1975, com a denominação de "Feira Municipal do Produtor", composta, na ocasião, por doze feirantes, regulamentada oficialmente pelo decreto municipal n.148/80, de 30 de outubro de 1980, com o nome de "Feira do Produtor Rural de Passo Fundo" (PASSO FUNDO, 1980).

Outro fator importante que concorre para a popularização das feiras é a crescente conscientização dos efeitos benéficos de uma dieta rica em frutas e hortaliças na saúde humana (CERDEÑO, 2006). Sabese, por exemplo, que o consumo insuficiente de frutas e hortaliças aumenta o risco de doenças crônicas não transmissíveis, como as cardiovasculares e alguns tipos de câncer, além de estar entre os dez fatores de risco que mais causam mortes e doenças em todo o mundo (JAIME, 2007). A preocupação com a aparência física, também, tem sido uma das razões para o consumo crescente de frutas e hortaliças.

Apesar do elevado e crescente volume de "hortifrutigranjeiros" ofertados à população, as quantidades consumidas ainda são insuficientes para atender às necessidades do corpo, representando menos da metade das necessidades nutricionais diárias (JAIME, 2007).

A Feira de Passo Fundo opera com um número excessivo de feirantes dentro de um mesmo ambiente, oferecendo restrita diversidade de produtos aos consumidores. O resultado dessa relação é uma reduzida escala de negócios, associada a práticas comerciais muitas vezes inadequadas, resultando em elevação de custos e diminuição de eficiência.

A Feira do Produtor de Passo Fundo foi criada há mais de duas décadas, no entanto não existem trabalhos relacionados aos aspectos sociais e econômicos dessa atividade. Assim, este estudo tem como objetivo traçar o perfil socioeconômico dos feirantes e consumidores e fornecer subsídios para o planejamento de ações que promovam o desenvolvimento socioeconômico dos produtores, além de, ao mesmo tempo, aumentar a satisfação dos consumidores.

\section{MATERIAL E MÉTODOS}

Os dados deste trabalho foram coletados na Feira do Produtor de Passo Fundo, que ocorre regularmente na Gare, antiga estação ferroviária de Passo Fundo. O município de Passo Fundo, com área de $780 \mathrm{~km}^{2}$, situa-se na região do Planalto Médio do Rio Grande do Sul, Brasil, e tem, atualmente, cerca de 190 mil habitantes.

$\mathrm{O}$ estudo foi realizado nos meses de agosto e setembro de 2006. Os dados foram coletados por meio de dois questionários, um aplicado aos 58 feirantes e outro, aos 471 consumidores que efetivamente realizaram compras nesses dois dias. Os questionários abrangeram questões referentes aos aspectos socioeconômicos dos entrevistados e produtos ofertados/comprados na feira, como hortaliças, frutas, grãos, produtos de origem animal e produtos artesanais. Foram estabelecidas as seguintes faixas de renda $(\mathrm{R} \$):<500,00 ; 500,00-1.000,00 ; 1.000,00$ $2.000,00 ; 2.000,00-3.000,00 ; 3.000,00-5.000,00 \mathrm{e}$ $>5.000,00$. A escolaridade registrada teve como base os níveis e graus do sistema educacional brasileiro (IBGE, 2008).

O levantamento serviu de base para caracterizar alguns aspectos socioeconômicos dos produtores, como idade, tamanho familiar, renda familiar, renda per capita, grau de escolaridade, idade e valor gasto pelos consumidores. A renda per capita foi calculada dividindo-se a renda familiar informada pelo produtor pelo número de membros da família.

O grau de escolaridade dos feirantes foi registrado com base nas seguintes categorias: nenhum - feirante que não completou o ensino fundamental; fundamental - feirante que concluiu o ensino fundamental; médio - feirante com o ensino médio completo; superior - feirante com curso superior concluído; pós-graduação - inclui o feirante com nível superior e que tenha completado algum curso de pósgraduação, lato sensu ou stricto sensu.

A análise estatística e a síntese gráfica e tabular dos dados foram executados com os programas computacionais CoStat (CoHort Software, 2003).

\section{RESULTADOS E DISCUSSÃO}

A idade dos feirantes e consumidores da Feira dos Produtores de Passo Fundo abrangeu várias faixas etárias (Figura 1). Entre os 58 feirantes 


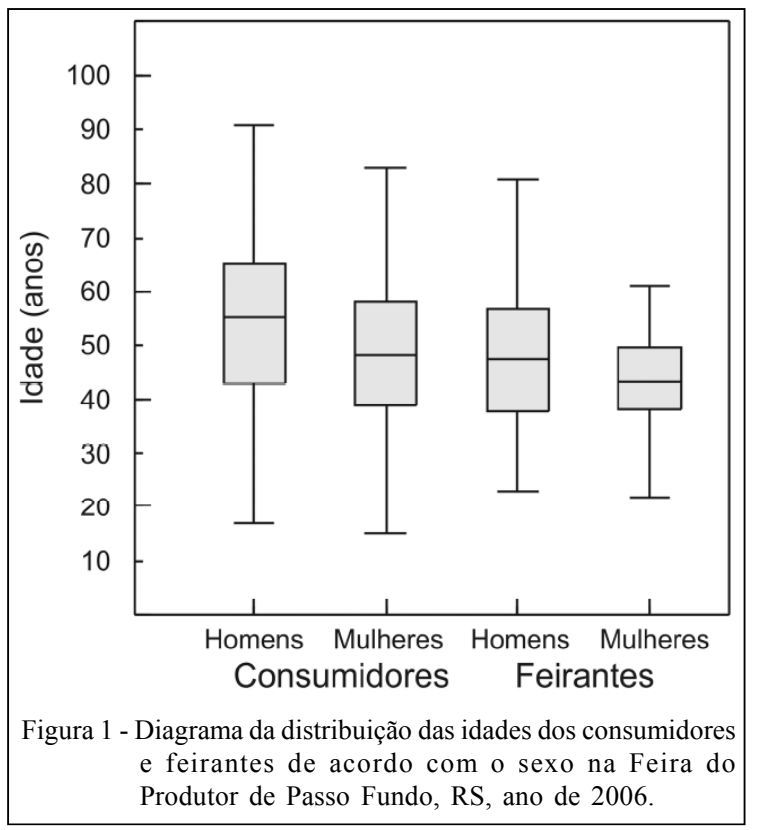

consultados pela pesquisa, 40 eram homens e 18 mulheres, a idade média correspondeu a 47 anos. Entre os 471 consumidores, 254 homens e 217 mulheres, a idade média foi de 52 anos.

Os homens frequentam com mais assiduidade a feira (51\%). Dos consumidores do sexo masculino, 50\% têm idade igual ou superior a 55 anos, o que sugere que o consumidor típico é um indivíduo, provavelmente, aposentado ou aproximando-se da aposentadoria. Esse dado se torna mais claro ao se verificar que $25 \%$ deles têm mais de 65 anos. Com relação às mulheres, aproximadamente $50 \%$ têm idade superior a 48 anos e 25\%, acima de 58 anos (Figura 1).

A composição familiar dos feirantes foi um dado importante deste estudo. Para um terço dos feirantes o núcleo familiar é composto por até três pessoas, o que sugere uma baixa disponibilidade de mão-de-obra para as atividades de produção de hortaliças e frutas na propriedade. Como a atividade de feirante é quase que estritamente familiar, o baixo número de membros das famílias envolvidas na produção de hortifrutigranjeiros pode se constituir em fator limitador da expansão dessa atividade em curto prazo e, até mesmo, levar ao desestímulo futuramente.

O maior percentual de feirantes do sexo masculino em todos os graus de educação, com exceção do nível superior, chama a atenção e contraria relatos de vários estudos sobre a educação e gênero no Brasil, os quais apontam, em geral, índices maiores de grau de alfabetização entre as mulheres (IBGE, 2000; FÍGOLI, 2006).
Ainda que relativamente baixos, os dados desta pesquisa mostram que quase $9 \%$ de feirantes possuem nível superior, mas não há registro de nenhum com o nível de pós-graduação. $\mathrm{O}$ grau de escolaridade mais comum entre os feirantes é o fundamental.

A renda mensal de quase $80 \%$ dos feirantes é de até $\mathrm{R} \$ 1.000,00$ (Figura 2). Um dos pressupostos deste estudo foi o de que feirantes de determinadas faixas de renda pudessem estar vinculados a certos tipos de produto, como tradicional, colonial ou orgânico, porém os resultados não evidenciaram essa relação.

O tamanho da família dos feirantes foi, também, examinado no estudo em associação com a renda média absoluta e per capita (Figura 3). Nesse aspecto, observou-se que, à medida que aumenta a família, a renda familiar tende a aumentar. Contrariamente à renda média familiar, a renda per capita diminui com o aumento do tamanho da família, de tal modo que os melhores resultados per capita são constatados em famílias menos numerosas. Verifica-se que há um decréscimo acentuado na renda per capita quando a família tem mais do que quatro pessoas, ao mesmo tempo em que a renda média familiar não aumenta. Várias podem ser as causas para esse fato, tais como a não-especialização da mão-de-obra, uma vez que se divide o tempo em diversas atividades concorrentes dentro da propriedade, e a pequena extensão da propriedade, o que impede a produção em escala econômica.

A Feira do Produtor de Passo Fundo é frequentada por consumidores de várias faixas de renda (Tabela 1). As rendas variam entre $\mathrm{R} \$ 500,00 \mathrm{e}$ $\mathrm{R} \$ 5.000,00$, com aproximadamente $60 \%$ deles declarando renda entre $\mathrm{R} \$ 500,00$ e $\mathrm{R} \$ 2.000,00$. Verificase que $26 \%$ dos consumidores homens e $26 \%$ das mulheres que frequentam a feira têm renda média mensal de até $\mathrm{R} \$ 1.000,00$. Dos consumidores com

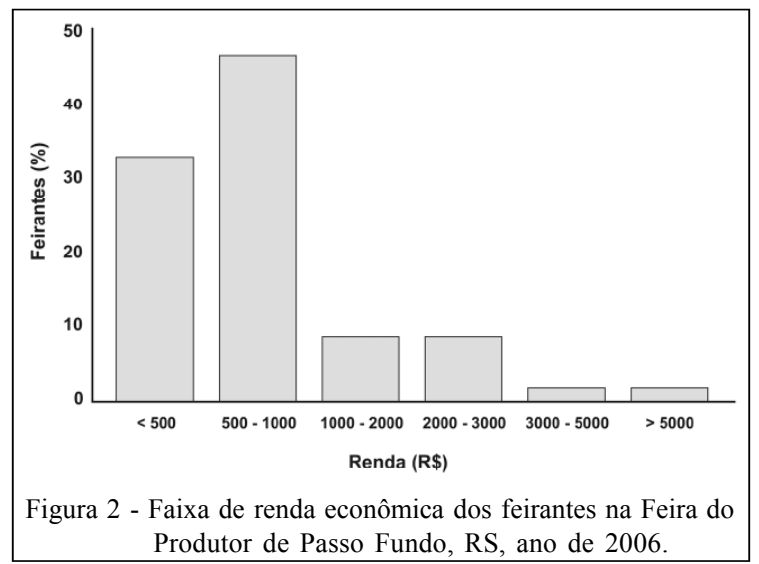




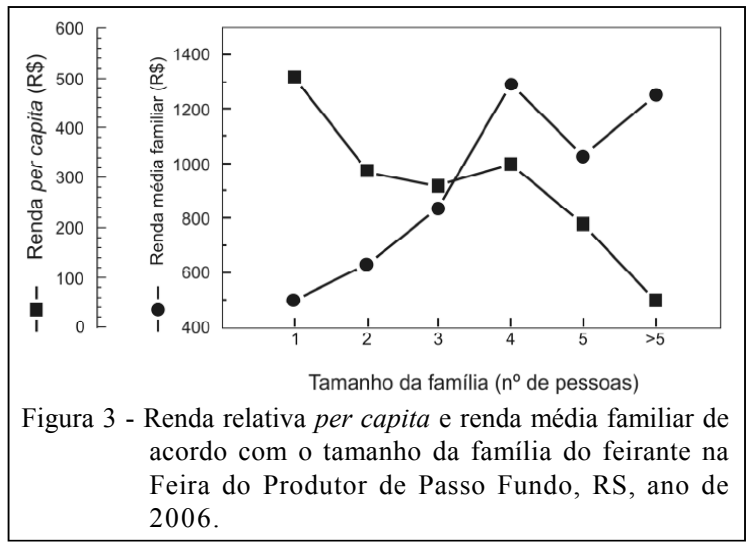

renda superior a $\mathrm{R} \$ 5.000,00,1,1 \%$ são mulheres e $2,1 \%$, homens.

Os gastos médios dos consumidores na Feira do Produtor de Passo Fundo variam conforme a faixa de renda (Tabela 1). O valor médio gasto pelos consumidores foi de $\mathrm{R} \$ 25,94 \mathrm{dia}^{-1}$, com os homens gastando, em média, $\mathrm{R} \$ 26,34 \mathrm{e}$ as mulheres, $\mathrm{R} \$ 25,48 \mathrm{dia}^{-1}$.

Os gastos observados mostram que, à medida que se eleva a faixa de renda, aumenta o consumo, o que concorda com o observado de modo geral no Brasil, onde o consumo está condicionado às condições socioculturais da população, ou seja, o crescimento nos níveis de consumo está associado à elevação da renda da população (IBGE, 2004).

A título de comparação, em nível internacional, o consumidor espanhol gastou em média, em 2005, $€ 115,3$ (R $\$ 298,23$ ) em frutas, $€ 84,4$ $(\mathrm{R} \$ 218,30)$ em hortaliças e $€ 24,3(\mathrm{R} \$ 62,85)$ em frutas e hortaliças transformadas, totalizando $€ 223$ ( $\mathrm{R} \$ 576,80)$, ao câmbio de $17 / 01 / 2008$, o que representa $17 \%$ do total gasto com alimentação. Os dados representaram uma demanda de $93,2 \mathrm{~kg}$ de frutas, $56,2 \mathrm{~kg}$ de hortaliças e $13,5 \mathrm{~kg}$ de frutas e hortaliças transformadas (CERDEÑO, 2006).

As feiras representam importante papel econômico tanto para produtores como para consumidores. Embora os consumidores vejam nas

Tabela 1 - Distribuição percentual do gênero e média de gasto dos consumidores da Feira dos Produtores de Passo Fundo, RS, de acordo com a faixa de renda, ano de 2006.

\begin{tabular}{lccc}
\hline $\begin{array}{l}\text { Faixa de renda } \\
(\mathrm{R} \$)\end{array}$ & $\begin{array}{c}\text { Feminino } \\
(\%)\end{array}$ & $\begin{array}{c}\text { Masculino } \\
(\%)\end{array}$ & $\begin{array}{c}\text { Média de gasto } \\
(\mathrm{R} \$)\end{array}$ \\
\hline$<500,00$ & 11,0 & 8,1 & 19,92 \\
$500,00-1000,00$ & 14,9 & 18,0 & 21,96 \\
$1000,00-2000,00$ & 12,1 & 13,6 & 25,33 \\
$2000,00-3000,00$ & 3,4 & 7,4 & 30,33 \\
$3000,00-5000,00$ & 3,6 & 4,7 & 32,89 \\
$>5000,00$ & 1,1 & 2,1 & 29,88 \\
\hline
\end{tabular}

feiras uma oportunidade de adquirir alimentos com preço baixo e com qualidade, o consumo de hortaliças no Brasil, avaliado em cerca de $29 \mathrm{~kg}$ per capita ${ }^{-1}$ ano $^{-1}$ (IBGE, 2004), é muito inferior ao verificado em outros países (RICHARDS \& PATTERSON, 2005; STATISTICS CANADA, 2004). No Rio Grande do Sul, entretanto, o consumo de frutas é de $67,1 \mathrm{~kg} \mathrm{hab}^{-1} \mathrm{ano}^{-1} \mathrm{e}$ de olerícolas, de $98,6 \mathrm{~kg} \mathrm{hab}^{-1}$ ano $^{-1}$, permitindo inferir um consumo de hortigranjeiros na ordem de $165,7 \mathrm{~kg} \mathrm{hab}^{-1}$ ano $^{-1}$ (DAMBORIARENA, 2001). Portanto, há grande potencial para o crescimento de feiras de produtores no país.

\section{CONCLUSÃO}

A Feira do Produtor de Passo Fundo é frequentada por consumidores de todos os níveis de escolaridade e faixas etárias, a maioria com idade entre 35 e 65 anos. Aproximadamente $80 \%$ dos feirantes têm renda inferior ou igual a $\mathrm{R} \$ 1.000,00$, ao passo que, dos consumidores, $75 \%$ têm renda igual ou inferior a $\mathrm{R} \$ 1.500,00$. Tamanhos familiares maiores determinam redução dos ganhos per capita dos feirantes, cujas causas podem ser a não especialização na atividade e o pequeno tamanho da propriedade, impedindo a produção em escala econômica.

Os gastos médios dos consumidores na feira variaram de $\mathrm{R} \$ 19,92$ a $\mathrm{R} \$ 32,89 \mathrm{dia}^{-1}$. Os homens são os consumidores mais frequentes da feira e seus gastos não diferem muito dos das mulheres.

\section{REFERÊNCIAS}

CERDEÑO, V.J.M. Hábitos de compra y consumo de frutas y hortalizas - Resultados del Observatório del Consumo y la Distribución Alimentaria. Rev Distribución y Consumo, n.88, p.5-28, 2006. Disponível em: http://www.mercasa.es/ nueva/revista/08_dyc_88.php. Acesso em: 9 jan. 2009.

CoHort Software. CoStat. Monterey, Califórnia. 2003. Disponível em: http://www.cohort.com. Acesso em: 10 jan. 2009.

DAMBORIARENA, E. Certificação e rotulagem na cadeia dos hortigranjeiros no estado do RS: um estudo de caso. 2001. 129f. CEASA/RS. Dissertação (Mestrado em Administração) - UFRGS, Porto Alegre, RS.

FÍGOLI, M.G.B. Evolução da educação no Brasil: uma análise das taxas entre 1970 e 2000 segundo o grau da última série concluída. R Bras Est Pop, v.23, n.1, p.129-150, 2006.

IBGE. Censo Demográfico, 2000. Disponível em: http:// www.ibge.gov.br/home/estatistica/populacao/censo2000/ default.shtm. Acesso em: 26 dez. 2008.

IBGE. Pesquisa de orçamentos familiares (POF), 2004. Disponível em: http://www.ibge.gov.br. Acesso em: 26 dez. 2008. 
JAIME, P.C. et. al. Educação nutricional e consumo de frutas e hortaliças: ensaio comunitário controlado. Rev Saúde Pública, [on line], v.41, n.1, p.154-157, 2007. Disponível em: http://www.scielosp.org. Acesso em: 12 jan. 2009.

PASSO FUNDO. Prefeitura Municipal Decreto $\mathbf{n}^{\mathbf{0}} \mathbf{1 4 8} / \mathbf{8 0}$ Aprova o regulamento da Feira do Produtor de Passo Fundo. 30 de outubro de 1980. 6p.
RICHARDS, T.J.; PATTERSON, P.M. A bilateral comparison of fruit and vegetable consumption: United States and Canada. J Agric Res Econ, v.30, n.2, p.333-349, 2005.

STATISTICS CANADA. Food consumption, 2004. Disponível em: http://www.statcan.gc.ca/dailyquotidien/051018/dq051018e-eng.htm. Acesso em: 12 jan. 2009 . 